\title{
Building and Breaking Interfaces: How a Receptor Takes Shape
}

\author{
Charu Chaudhry, ${ }^{1}$ Annalisa Scimemi, ${ }^{2}$ and Janesh Kumar ${ }^{1}$ \\ ${ }^{1}$ Laboratory of Cellular and Molecular Neurophysiology, National Institute of Child Health and Human Development, and ${ }^{2}$ Synaptic Physiology \\ Section, National Institute of Neurological Disorders and Stroke, Porter Neuroscience Research Center, National Institutes of Health, Bethesda, \\ Maryland 20892-3701 \\ Review of Farina et al.
}

Most ion channels and neurotransmitter receptors assemble into functional heterooligomers with precise stoichiometry and composition. Ionotropic glutamate receptors (GluRs), for example, the main mediators of excitatory neurotransmission, are either obligatory NMDA (GluN1-3) or preferential AMPA (GluA1-4) and kainate (GluK1-5) heterotetramers. The extracellular N-terminal domain (NTD) and ligandbinding domain (LBD) of each subunit interact with partner subunits at the level of both the NTD and LBDs to form a dimerof-dimers in the full-length tetrameric receptor (Mayer, 2011). How different subunit pairs are incorporated and others excluded during receptor biogenesis remains largely unresolved, despite the profound consequences that proper ionotropic GluR assembly has for neuronal physiology and pathology. Critical spatiotemporal parameters include distinct subunit expression profiles, local concentrations during initial synthesis in the endoplasmic reticulum (ER) and subsequent diffusion, and relative affinities between subunits.

Recent structural and biophysical studies have explored the role of the NTDs in controlling ionotropic GluR subunit assembly. In a recent issue of The Journal of Neuroscience, Farina et al. (2011) show how distinct NTD properties of NMDA receptors, which are arguably the most complex ionotropic GluRs, affect assembly. They identify a novel biosynthetic intermediate that leads to new speculations on how these receptors are assembled in cells.

Most GluNs consist of two glycinebinding GluN1 subunits and two glutamatebinding GluN2 subunits. Farina et al. (2011) first show that GluN2 requires GluN1 to traffic to the cell surface: GluN2 is only present on the cell surface when coexpressed with wild-type GluN1, but not with an NTD-lacking GluN1 (GluN1 $\Delta$ NTD). Remarkably, coexpression of isolated GluN1-NTD along with GluN1 $\Delta$ NTD rescues surface expression of GluN2A. The isolated GluN1-NTD also rescues expression of GluN2A/B-NTD, as a folded soluble protein secreted from the ER, presumably through a direct physical interaction [Farina et al. (2011), their Fig. 1]. Whether the rescued surface expression of GluN2A results in production of a functional receptor remains to be tested with electrophysiological recordings.

What happens to receptor assembly if the NTD heterodimer is stabilized? The authors scan for mutations in GluN1-NTD that enhance heterodimerization with the GluN2-NTD and promote its surface expression, using NTD dimer structures of GluA2 and GluK2 as guides. They identify
GluN1-NTD-T110A as a mutation that promotes cosecretion and coimmunoprecipitation of GluN2-NTD. Does GluN1NTD-T110A also have a greater ability to rescue GluN2A-GluN1 $\Delta$ NTD over wildtype GluN1-NTD, and accelerate heteromeric assembly? When the authors test this in intact receptors, they see a strikingly opposite result: a decrease in receptor surface expression. Thus, assembly involves more complex mechanisms than simple initial heterodimerization. What are the NTD affinities that drive GluN1-GluN1 homomer versus GluN1-GluN2 heteromer formation? Direct measurements of these affinities, which are critical to understanding the different assembly pathways, need to be performed using biophysical methods (e.g., analytical ultracentrifugation).

Interestingly, when Farina et al. (2011) solve the structure of GluN1-NTD, it crystallizes as a dimer. Each clamshell-shaped protomer has a similar $\sim 45^{\circ}$ twist in the lower lobe (L2) as previously observed in the GluN2B-NTD monomer structure. The dimer packing seen for GluN1-NTD is unique because all the contacts are mediated by the upper lobe (L1) and the GluN1-NTD has a twist that is absent in GluA2 and GluK2, such that the L2 domains are oriented in opposite directions relative to L1 [Farina et al. (2011), their Fig. 5]. This arrangement presumably allows GluN-NTDs to undergo changes in domain closure in response to allosteric ligands that can modulate channel activ- 
ity. The GluN1 dimer interface at L1 is mediated by helix $\alpha 3$ from both protomers, which stack onto each other, while helix $\alpha 2$ interacts with a novel helix $\alpha 1$ absent in GluA/GluK. In contrast, the L1 interface in the NTDs of GluA and GluK is mediated by residues from helix $\alpha 2$ stacking onto helix $\alpha 3$ in a twofold symmetric manner [Farina et al. (2011), their Fig. 6]. This key difference in dimer packing reflects alterations in how the L1 interface is constructed in different ionotropic GluRs.

As is important whenever interpreting crystal structures, the authors ask whether the dimer packing they observe is physiological. Using cysteine cross-linking and a Y109C mutation on helix $\alpha 3$ that makes spontaneous dimers from both isolated NTD and full-length GluN1, they test whether the homodimer initially formed by GluN1 subunits during receptor assembly remains intact in mature GluNs, or whether instead the GluN1 homodimer serves as an assembly intermediate that reorganizes during tetramerization. If the latter is true, constraining the GluN1-NTD interface should stall assembly. To test this, both GluN1-Y109C and GluN2B were coexpressed. When GluN1-Y109C was then coimmunoprecipitated with GluN2, only a small fraction of GluN1-Y109C crosslinked with GluN2, indicating that forced dimerization of GluN1-NTD is unfavorable in the mature heterotetramer and, consequently, that the homodimer may be an assembly intermediate. Does a GluN1 homodimer form along the assembly pathway? Visualization of biosynthetic intermediates using single-particle electron microscopy, as performed for GluA (Shanks et al., 2010), would provide direct structural proof for the transient GluN1 homodimer. It is worth noting that earlier FRET experiments also detected GluN1 homodimers (Qiu et al., 2005), and in another recent study GluN1 was seen to form weak spontaneous cross-linked homodimers during biogenesis, based on whole-cell Western blots (Lee and Gouaux, 2011).

Based on these results, a plausible hypothesis is that separation of GluN1 dimers is required for GluN2 incorporation into heterotetramers. The authors provide proof for GluN1-GluN2 subunit exchange by cotransfecting a fixed amount of GluN1-NTD-Y109C with increasing amounts of GluN2-NTD, and showing a concentration-dependent inhibition of GluN1 homodimerization by GluN2. The same result is recapitulated using full-length subunits, underscoring that GluN2-NTD competitively associates with GluN1-NTD, driving rearrangements within the intact re-
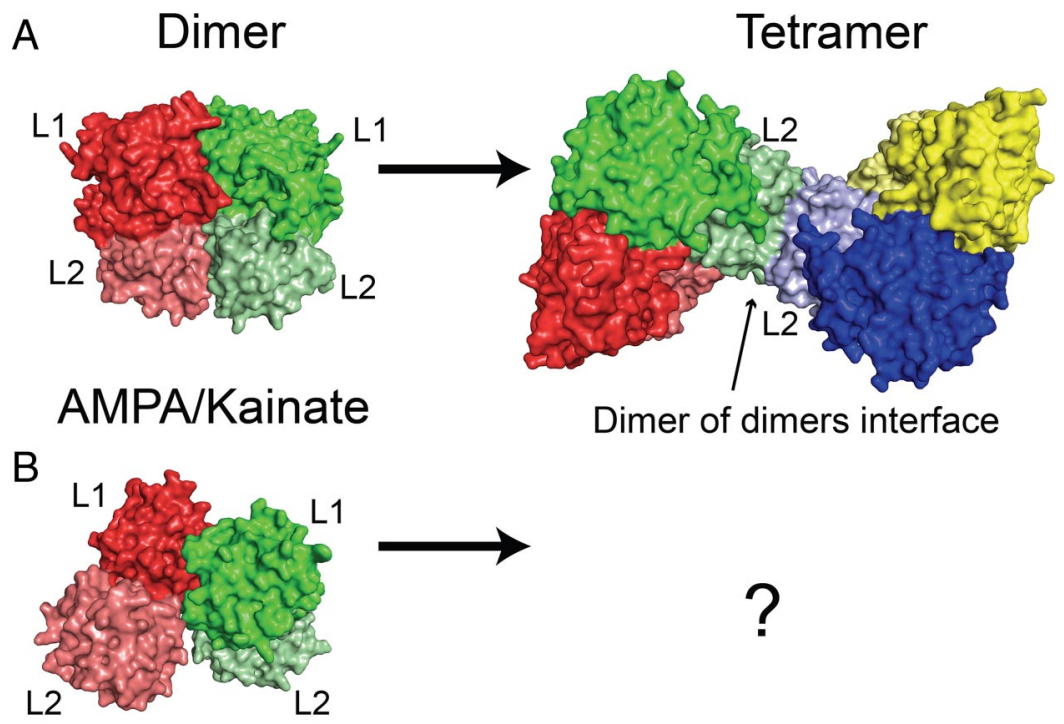

NMDA

Figure 1. N-terminal domains of ionotropic GluRs in tetrameric receptor assembly. $\boldsymbol{A}$, Left, Structure reflects the NTD dimer conformation in an AMPA/kainate receptor (PDB codes 3KG2, 3H6G). Each subunit forms a bilobed clamshell composed of two halves, $\mathrm{L} 1$ and $\mathrm{L} 2$, with extensive intersubunit contacts (L1-L1 and L2-L2). Right, Structure of the interface between two NTD dimers in the tetrameric receptor. The interface is located on a twofold symmetry axis, and is mediated by the L2 lobes of the two proximal subunits. $\boldsymbol{B}$, Structure of the GluN-NTD dimer (PDB code 3041). Each subunit also forms a bilobed structure (as in $\boldsymbol{A}$, left) but with a twisted L1-L2 conformation. The intersubunit contacts within the dimer are mediated by the L1 lobes only, leaving unanswered how two NTD dimers pack in the tetramer (right). The four subunits in the tetramer assembly are colored red, green, yellow, and blue; the L1 and L2 lobes are in dark and light shades, respectively.

ceptor. To further test this, Farina et al. (2011) resort to their earlier cellular trafficking assay substituting GluN1-NTD-Y109C for GluN1-NTD to see whether the trafficking defect of GluN2A-GluN1 $\Delta$ NTD could be rescued. If GluN1 dimer separation is required for efficient heteromeric assembly, there should be decreased rescue relative to wild type. This is what they observe.

The effects of the T110A and Y109C mutations on assembly suggest that efficient heteromeric assembly requires a dimer system carefully poised between homomerization and heteromerization. A remaining question is how the NTDs have evolved to achieve this balance. Previous studies indicate that GluN1 is expressed as a monomer/dimer and is relatively stable, whereas GluN2 tends to aggregate and more rapidly degrade, but increases its stability when coexpressed with GluN1 (Atlason et al., 2007). Does GluN2 undergo a conformational change or fold upon binding to GluN1? A crystal structure of the GluN1-GluN2 NTD heterodimer compared to the GluN2-NTD monomer structure would elucidate relevant conformational changes on heteromerization. Also, thermodynamic studies of GluN1-GluN2 NTD interactions should be informative, particularly analysis of enthalpic and entropic contributions to binding; for example, changes in heat capacity may discriminate between rigid body association and coupled conformational changes. Other examples of coupled folding/binding events have been reported, as in the case of nicotinic acetylcholine receptor assembly (Wanamaker et al., 2003).

In conclusion, the experiments by $\mathrm{Fa}-$ rina et al. (2011) suggest a sequential assembly model in which GluN2 monomers add to a GluN1 homodimeric intermediate, which reequilibrates into a GluN1-GluN2 dimer-of-dimers during GluN biogenesis. The mystery is why the metastable GluN1GluN1 homodimer, as opposed to an initial GluN1-GluN2 heterodimer, is required along the assembly pathway. One hypothesis is that it enables correct docking of GluN2 and "induced-fit" of GluN1 and GluN2 to form the native heterotetramer, but the molecular interplay between domains of the two subunits remains elusive. Notably, this model is distinct from heteromeric GluA assembly, where biophysical and electrophysiology studies suggest that heteromers form at the initial dimer level (Rossmann et al., 2011). Is the GluN tetramer then different from the GluA one? First, the recently solved GluA2 structure shows that subunit pairs that form NTD dimer assemblies swap partners in the LBD (Sobolevsky et al., 2009), 
but whether this occurs in GluN is not clear. Using the GluN1-NTD homodimer structure and recently reported GluN1GluN2 NTD cross-links (Lee and Gouaux, 2011) together with the previously solved LBD GluN1-GluN2 heterodimer structure as guides, cross-link pairs can be designed to test for domain swapping. Second, the twisted conformation of GluN-NTDs leads to a difference in NTD dimer assembly relative to GluA/GluKNTDs, and implies that the tetramerization interface is also different in GluN (i.e., weaker) (Fig. 1). This idea is supported by recent cross-linking experiments by Lee and Gouaux (2011) showing that GluN1 and GluN2-NTDs form local heterodimers in the mature receptor through L1-L1 and not L2-L2 interactions, in contrast to GluA/GluK-NTDs where both lobes can be cross-linked.

So what is the primary energy driving force for GluN tetramerization and how is the energetic cost for GluN1 homodimer disassembly compensated? Possible answers may include additional folding enthalpy of GluN2-NTD on binding GluN1, along with interactions at the LBD and also transmembrane and C-terminal domains (CTDs). Notably, GluNs have ex- tensive CTDs that distinguish them from other ionotropic GluRs. Could these contribute additional stabilization to the tetramer through interaction with each other or other proteins, thus compensating for weaker NTD associations?

Other questions also remain, such as, what is the efficiency of assembly, and is it regulated? It is tempting to speculate that binding of allosteric ligands to the NTD pocket may modulate the assembly process, much like binding of agonist to the LBD cleft influences gating. The association of GluNs with auxiliary proteins, such as NETO1 that interacts with GluN2 subunits, may provide another unexplored level of complexity influencing biosynthesis. The work by Farina et al. (2011), which places the dynamic GluNNTDs at center stage of the structural and functional landscape of the receptor, will undoubtedly catalyze new hypotheses for future studies.

\section{References}

Atlason PT, Garside ML, Meddows E, Whiting P, Mcllhinney RA (2007) N-Methyl-D-aspartate (NMDA) receptor subunit NR1 forms the substrate for oligomeric assembly of the NMDA receptor. J Biol Chem 282:25299-25307.

Farina AN, Blain KY, Maruo T, Kwiatkowski W,
Choe S, Nakagawa T (2011) Separation of domain contacts is required for heterotetrameric assembly of functional NMDA receptors. J Neurosci 31:3565-3579.

Lee CH, Gouaux E (2011) Amino terminal domains of the NMDA receptor are organized as local heterodimers. PLoS One 6:e19180.

Mayer ML (2011) Structure and mechanism of glutamate receptor ion channel assembly, activation and modulation. Curr Opin Neurobiol 21:283-290.

Qiu S, Hua YL, Yang F, Chen YZ, Luo JH (2005) Subunit assembly of N-methyl-d-aspartate receptors analyzed by fluorescence resonance energy transfer. J Biol Chem 280:24923-24930.

Rossmann M, Sukumaran M, Penn AC, Veprintsev DB, Babu MM, Greger IH (2011) Subunitselective $\mathrm{N}$-terminal domain associations organize the formation of AMPA receptor heteromers. EMBO J 30:959-971.

Shanks NF, Maruo T, Farina AN, Ellisman MH, Nakagawa T (2010) Contribution of the global subunit structure and stargazin on the maturation of AMPA receptors. J Neurosci 30:2728-2740.

Sobolevsky AI, Rosconi MP, Gouaux E (2009) $\mathrm{X}$-ray structure, symmetry and mechanism of an AMPA-subtype glutamate receptor. Nature 462:745-756.

Wanamaker CP, Christianson JC, Green WN (2003) Regulation of nicotinic acetylcholine receptor assembly. Ann N Y Acad Sci 998:66-80. 